\title{
STRUCTURE SETS VANISH FOR CERTAIN BUNDLES OVER SEIFERT MANIFOLDS \\ BY
}

\author{
CHRISTOPHER W. STARK
}

\begin{abstract}
Let $M^{n+3}$ be a compact orientable manifold which is the total space of a fiber bundle over a compact orientable manifold $K^{3}$ with an effective circle action of hyperbolic type. Assume that the fiber $N^{n}$ in this bundle is a closed orientable manifold with Noetherian integral group ring, with vanishing projective class and Whitehead groups, and such that the structure set $S_{\text {TOP }}\left(N^{n} \times D^{k}, \partial\right)$ of topological surgery vanishes for sufficiently large $k$. Then the projective class and Whitehead groups of $M$ vanish and $S_{\text {TOP }}\left(M^{n+3} \times D^{k}, \partial\right)=0$ if $n+k \geqslant 3$ or if $K^{3}$ is closed and $n=2$. The UNil groups of Cappell are the main obstacle here, and these results give new examples of generalized free products of groups such that $\mathrm{UNil}_{j}$ vanishes in spite of the failure of Cappell's sufficient condition.
\end{abstract}

A conjecture of long standing holds that a closed aspherical manifold is determined up to homeomorphism by its fundamental group. This claim is now known to be valid for aspherical manifolds of dimensions greater than four whose fundamental groups contain nilpotent subgroups of finite index [8,10]; earlier work verified the conjecture for poly-Z fundamental groups, subject to the same dimension restrictions [30, pp. 228-231].

This paper will show that the conjecture and related statements are valid for compact manifolds $M$ which are total spaces of certain bundles over surfaces or 3-manifolds. The fibers of these bundles are closed, orientable manifolds $N$ satisfying these two hypotheses:

HyPOTHESIS A. $\mathbf{Z} \pi_{1} N$ is a right regular Noetherian $\operatorname{ring}, \operatorname{Wh}\left(\pi_{1} N\right)=0$, and $K_{0}\left(\mathbf{Z} \pi_{1} N\right)=0$.

HYPOTHESIS B. $S_{\text {TOP }}\left(N \times D^{k}, \partial\left(N \times D^{k}\right)\right)=0$ if $k$ is large enough. Here $S_{\text {TOP }}(-)$ is the structure set of topological surgery [15] and the conjecture is verified by showing that $S_{\text {TOP }}(M, \partial M)$ vanishes for the manifolds under study. Note that if $N$ is aspherical and $\pi_{1} N$ is poly-Z or virtually nilpotent, then both hypotheses are satisfied, so there is a large supply of suitable fibers, including such classes of interest as closed Riemannian flat manifolds.

Received by the editors May 2, 1983.

1980 Mathematics Subject Classification. Primary 57R65; Secondary 18F25.

Key words and phrases. Seifert manifold, fiber bundle, algebraic $K$-theory, projective class group, Whitehead group, surgery, structure set, splitting theorems, UNil. 
Most of the 3-manifolds to be considered here are aspherical, orientable, 3-manifolds $K^{3}$ with circle actions such that $K^{3} / S^{1}$ is an orientable surface, and the use of the term "Seifert manifold" will be confined to such 3-manifolds. The main result of this paper is this:

THEOREM. Let $N^{n}$ be a closed, connected, orientable manifold which satisfies Hypotheses A and B. $\left(N=\{\right.$ point $\}$ is permitted.) Let $M^{n+3}$ be the orientable total space of a bundle over $K^{3}$ with fiber $N^{n}$, where $K^{3}$ is a compact, connected, aspherical, orientable 3-manifold such that either

(a) $K^{3}$ is a Haken manifold fibering over $S^{1}$,

(b) $K^{3}$ is a Seifert manifold with nonempty boundary,

(c) $K^{3}$ is a closed Seifert manifold such that genus $\left(K^{3} / S^{1}\right) \geqslant 1$,

(d) $K^{3}$ is a closed Seifert manifold such that $K^{3} / S^{1}=S^{2}$, every isotropy subgroup for the circle action on $K^{3}$ has odd order, and the group $Q\left(\alpha_{1}, \alpha_{2}, \ldots, \alpha_{m}\right)=\pi_{1} K^{3} / \pi_{1} S^{1}$ is Fuchsian, or

(e) $K^{3}=M\left(b ; 0,0 ;\left(\alpha_{1}, \beta_{1}\right), \ldots,\left(\alpha_{m}, \beta_{m}\right)\right)$, where an odd prime $p$ divides $\alpha_{1}$ and the group $Q\left(\alpha_{1} / p, \alpha_{2}, \ldots, \alpha_{m}\right)$ is Fuchsian.

Then $S_{\mathrm{TOP}}\left(M^{n+3} \times D^{k}, \partial\left(M \times D^{k}\right)\right)=0$ if $n+k \geqslant 3$ or if $\partial K=\varnothing, k=0$, and $n=2$.

See $\$ 1$ for the language used in stating (d) and (e). Recall that a Fuchsian group is a discrete subgroup of the isometry group of the hyperbolic plane $\mathbf{R} H^{2}$.

The first section of this paper gives vanishing results for the projective class and Whitehead groups of our manifolds $M$, obtained by combining Waldhausen's results and Frobenius induction. A new proof of the main lemma of [19] is given here. $\$ 2$ discusses the apparatus for structure set computations, obtains the analog of the main theorem for bundles over surfaces, and proves (a), (b), (c) and part of (d). The keystone of the paper is the argument for (c), which concludes $\$ 2$. The third section deals with cases in which $K^{3} / S^{1}=S^{2}$ by Frobenius induction, reducing them to manifolds already analyzed, and here (e) and the rest of (d) are considered.

Cappell's UNil groups are the main difficulty to overcome in studying these manifolds and the vanishing results proved here show that in many instances where Cappell's sufficient condition [2,4] for the vanishing of UNils fails they are zero nonetheless. The result above falls a little short of what one expects-it should be true that any Seifert manifold $K^{3}$ which is sufficiently large or which has a sufficiently large finite-sheeted covering space admits such a vanishing theorem, proved by the tools used here. The work of Farrell and Hsiang fills in some of the omissions above (those in which $Q\left(\alpha_{1}, \alpha_{2}, \ldots, \alpha_{m}\right)$ is a group of Euclidean isometries and so contains proper subgroups isomorphic to itself), at the cost of further restrictions on the fiber $N$, by studying expanding maps. The other omissions reduce to the study of $K^{3}$ 's with circle actions whose isometry groups are all 2-primary. It seems curious that, because of difficulties in carrying out Frobenius induction, the 2-primary UNil groups have been most intran sigent when these isometry groups are also 2-primary. 
This paper is drawn from the author's dissertation for the University of Michigan [25], and he wishes to thank Tom Farrell, who supervised this research, and Frank Raymond. Both were constantly encouraging and helpful. Andrew Nicas and Andrew Ranicki were most kind in offering preprints and correspondence concerning unpublished work.

1. Whitehead and projective class groups. The techniques of Waldhausen $[28,29]$ for computations in $K$-theory work best under certain regularity hypotheses on the group rings under study. Recall that a ring is called right coherent if its finitely presented right modules form an Abelian category. Note that any right Noetherian ring is right coherent. A ring is called right regular if each of its finitely generated right modules has a finite-dimensional projective resolution. A group ring $\mathbf{Z} G$ is right regular if $G$ has finite cohomological dimension, so all groups $G$ with finite $K(G, 1)$ complexes have right regular integral group rings. Waldhausen's Nil group for the free product with amalgamations $G=A{ }_{C} B$ or the HNN construction $G=A{ }_{C}$ vanishes if $\mathbf{Z C}$ is right regular coherent; this leads to a Mayer-Vietoris sequence for the $K$-theory of $G$ [28]. Results in the cited papers of Waldhausen and in Farrell and Hsiang [7] lead to vanishing results for the Whitehead and projective class groups of the class of manifolds under study in this paper if we make the following assumptions concerning the manifolds which will serve as fibers:

HyPOTHESIS A. $\mathbf{Z} \pi_{1} N$ is a right regular Noetherian ring and $\mathrm{Wh}\left(\pi_{1} N\right)=$ $\tilde{K}_{0}\left(\mathbf{Z} \pi_{1} N\right)=0$.

Familiar examples of manifolds which satisfy these conditions include those with poly-Z fundamental groups and the complete Riemannian flat manifolds.

LEMMA 1.1. Let $N^{n} \rightarrow M^{n+k} \rightarrow^{p} K^{k}$ be a fiber bundle where $N$ is a connected manifold satisfying Hypothesis A. If $K$ is a (possibly bounded or noncompact) surface other than $S^{2}$ or $\mathbf{R} P^{2}$ then $\mathbf{Z} \pi_{1} M$ is a right regular ring and $\mathrm{Wh}\left(\pi_{1} M\right)=\tilde{K}\left(\mathbf{Z} \pi_{1} M\right)$ $=0$.

If $K$ is a Haken 3-manifold then $\mathrm{Wh}\left(\pi_{1} M\right)=\tilde{K}_{0}\left(\mathbf{Z} \pi_{1} M\right)=0$.

PROOF. A surface other than $S^{2}$ or $\mathbf{R} P^{2}$ can be cut open along imbedded circles and arcs to obtain a disjoint union of 2-discs. Take the preimages under $p$ of such a sequence of circles and arcs to give $\pi_{1} M$ a "graph of groups" or "generalized free product" structure in which all the amalgamations are made along copies of $\pi_{1} N$ or along extensions of the infinite cyclic group $T$ by $\pi_{1} N$ obtained by restricting the bundle to one of the imbedded circles in its base. In either case, Hypothesis A guarantees that we are amalgamating over a right regular Noetherian group ring so that Waldhausen's Nil group vanishes, and induction using Waldhausen's MayerVietoris sequence as we assemble the bundle and its fundamental group with this graph of groups structure gives the result that $\mathrm{Wh}\left(\pi_{1} M\right)=\tilde{K}_{0}\left(\mathbf{Z} \pi_{1} M\right)=0$. The regularity of $\mathbf{Z} \pi_{1} M$ follows from a result of Waldhausen: if $G$ has a graph of groups structure in which the building blocks have right regular coherent group rings and the amalgamations have right regular Noetherian group rings then $Z G$ is right regular coherent. 
The same tools yield the second claim: induce over a hierarchy of 3-manifolds obtained by cutting open $K$ along incompressible surfaces, using the same results of Waldhausen. This completes the proof of 1.1 .

Frobenius reciprocity and induction theorems for $K$-theory [26, 27] yield vanishing results for some non-Haken 3-manifolds with finite-sheeted covers which are Haken (these are now known as “virtually Haken" manifolds- see [13]). An argument of this sort is given in Plotnick's paper [19] and will be generalized here.

This paper is concerned with orientable Seifert manifolds $K^{3}$ over orientable surfaces (which may have nonempty boundary), where the Seifert fibered structure is given by a fixed-point free circle action on $K^{3}$. We adopt a modified version of Orlik's notation in [18] to write

$$
K^{3}=M\left(b ; g, d ;\left(\alpha_{1}, \beta_{1}\right),\left(\alpha_{2}, \beta_{2}\right), \ldots,\left(\alpha_{m}, \beta_{m}\right)\right)
$$

if $K^{3}$ is the Seifert manifold whose base is the orientable surface of genus $g$ with $d$ boundary components, whose $i$ th exceptional orbit has isotropy group $T_{\alpha_{i}}$ (the cyclic group of order $\left.\alpha_{i}\right)$ and winding invariant $\beta_{i}\left(0<\beta_{i}<\alpha_{i}\right)$, and cross-section obstruction $b \in Z$. See [18] for details on $b$ and $\beta_{i}$; note that $b$ is not needed if $K$ has nonempty boundary, so when $d>0$ we replace $b$ by a dash. These numerical invariants determine $K^{3}$ but are not unique. The Seifert manifolds with three exceptional fibers over $S^{2}$,

$$
K^{3}=M\left(b ; 0,0 ;\left(\alpha_{1}, \beta_{1}\right),\left(\alpha_{2}, \beta_{2}\right),\left(\alpha_{3}, \beta_{3}\right)\right)
$$

with $\alpha_{1}^{-1}+\alpha_{2}^{-1}+\alpha_{3}^{-1} \leqslant 1$ are the best-understood virtually Haken 3-manifolds, and for those with $\alpha_{1}^{-1}+\alpha_{2}^{-1}+\alpha_{3}^{-1}<1$ Plotnick showed that $\mathrm{Wh}\left(\pi_{1} K\right)=\tilde{K}_{0}\left(\mathbf{Z} \pi_{1} K\right)=$ 0 . His work was based on the next lemma, for which we give a new proof that does not require an analysis of the permutations in Fox's paper [11].

Recall that a finite group $G$ is p-elementary if it is the direct product of a cyclic group and a $p$-group, $G=T_{k} \times P$, where $T_{k}$ is the cyclic group of order $k$ and $P$ is a $p$-group (here $p$ is any prime); a finite group is elementary if it is $p$-elementary for some prime $p$. A finite group $G$ is $p$-hyperelementary if it is a semidirect product $G=T_{k} \rtimes P$ of a cyclic group and a $p$-group; $G$ is hyperelementary if it is $p$-hyperelementary for some prime $p$.

LEMMA 1.2. Let $K^{3}$ be the Seifert manifold $M\left(b ; 0,0 ;\left(\alpha_{1}, \beta_{1}\right),\left(\alpha_{2}, \beta_{2}\right),\left(\alpha_{3}, \beta_{3}\right)\right)$, where $\alpha_{1}^{-1}+\alpha_{2}^{-1}+\alpha_{3}^{-1} \leqslant 1$. Let $j: S^{1} \hookrightarrow K^{3}$ be the inclusion of a nonexceptional fiber and let $\mathbf{Q}=\pi_{1} K / j_{\#}\left(\pi_{1} S^{1}\right)$ be the quotient group associated to this Seifert fibered structure. Then $\mathbf{Q}$ has a finite quotient group which is not hyperelementary.

Proof. $Q=Q\left(\alpha_{1}, \alpha_{2}, \alpha_{3}\right)$ has the presentation $Q=\left\langle q_{1}, q_{2}, q_{3}: q_{1}^{\alpha_{1}}=q_{2}^{\alpha_{2}}=q_{3}^{\alpha_{3}}\right.$ $\left.=q_{1} q_{2} q_{3}=1\right\rangle$, so it is isomorphic to the orientation-preserving subgroup of a Schwarz triangle group. $Q$ has a normal, torsion-free subgoup $S$ of finite index, and $S$ is isomorphic to the fundamental group of a closed surface of genus greater than 0 . There are at least three ways to establish the existence of such a subgroup $S$. The oldest argument is Fox's [11], in which explicit homomorphisms to permutation groups are constructed whose kernels will serve as $S$. Given the fact that such a group can be realized as a group of isometries of the hyperbolic or Euclidean plane 
then the existence of $S$ can be established in the hyperbolic case by showing that finitely generated matrix subgroups of $\operatorname{SL}(n, F)(n \geqslant 1, F=$ any field) are residually finite (see [16, pp. 143-145]) and Bieberbach's theorems on crystallographic groups yield the flat case. Hempel's text [13] gives a third proof using the theory of covering spaces (pp. 118-120).

Such a subgroup $S$ gives us an extension $1 \rightarrow S \rightarrow Q \rightarrow G \rightarrow 1$, where $G$ is a finite group. Let $g=\operatorname{genus}(S) \geqslant 1$. Let $r$ be the product of two distinct primes $s$ and $t$, let $T_{r}$ be the cyclic group of order $r$, and let $T$ be the infinite cyclic group. The Abelianization homomorphism gives a composite $S \rightarrow T^{2 g} \rightarrow\left(T_{r}\right)^{2 g}$; let $N$ be the kernel of its composite epimorphism. It is not hard to see that $N$ is a characteristic subgroup of $S$, and hence normal in $Q$, so that we can consider the extension

$$
\begin{aligned}
& 1 \rightarrow S / N \rightarrow \underset{\|}{Q / N} \rightarrow G \rightarrow 1 . \\
& \left(T_{r}\right)^{2 g}
\end{aligned}
$$

Whether or not $G$ is hyperelementary, the finite group $Q / N$ cannot be hyperelementary. To see this, not that any subgroup of $p$-hyperelementary group must be $p$-hyperelementary, while $S / N=\left(T_{r}\right)^{2 g}=\left(T_{s}\right)^{2 g} \times\left(T_{t}\right)^{2 g}$ (recall that $s$ and $t$ are distinct primes) is $p$-hyperelementary for no prime $p$. This completes the proof of 1.2.

The following induction theorem is a consequence of Swan's work [26, 27]. The analogous statement holds for $\tilde{K}_{0}$.

Proposition 1.3. Let $\pi$ be a group with an epimorphism $f: \pi \rightarrow G$, where $G$ is a finite group. Let $x \in \mathrm{Wh}(\pi) . x=0$ if and only for each hyperelementary subgroup $H$ of $G, i_{H}^{*}(x)=0$ in $\mathrm{Wh}\left(f^{-1}(H)\right)$, where $i_{H}^{*}: \mathrm{Wh}(\pi) \rightarrow \mathrm{Wh}\left(f^{-1}(H)\right)$ is the transfer homomorphism.

This has been applied in computations for Bierbach groups by Farrell and Hsiang $[8,9]$ and by Plotnick to the insufficiently large Seifert manifolds

$$
M\left(b ; 0,0 ;\left(\alpha_{1}, \beta_{1}\right),\left(\alpha_{2}, \beta_{2}\right),\left(\alpha_{3}, \beta_{3}\right)\right) \text { with } \alpha_{1}^{-1}+\alpha_{2}^{-1}+\alpha_{3}^{-1}<1 .
$$

The next proof follows Plotnick's argument.

Proposition 1.4. Let $K^{3}=M\left(b ; 0,0 ;\left(\alpha_{1}, \beta_{1}\right),\left(\alpha_{2}, \beta_{2}\right),\left(\alpha_{3}, \beta_{3}\right)\right)$ with $\alpha_{1}^{-1}+\alpha_{2}^{-1}$ $+\alpha_{3}^{-1}<1$. Let $N^{n} \rightarrow M^{n+3} \rightarrow K^{3}$ be a fiber bundle over $K$ with a fiber that satisfies Hypothesis A. Then $\mathrm{Wh}\left(\pi_{1} M\right)=\tilde{K}_{0}\left(\mathrm{Z} \pi_{1} M\right)=0$.

Proof. These remarks will treat only the Whitehead group as the arguments for Whand $\tilde{K}_{0}$ are the same. 1.3 and 1.1 show that it would suffice to take a nonhyperelementary finite quotient $G$ of $\pi_{1} K=\pi_{1} M / \pi_{1} N$ by invoking 1.2 and to show that each covering space $K_{H}$ of $K$ which corresponds to a hyperelementary subgroup $H$ of $G$ is Haken. While we cannot quite guarantee this, our difficulties are limited to the possibility that one Seifert manifold with three exceptional orbits over $S^{2}$ is covered by another Seifert manifold with three exceptional orbits over $S^{2}$. In terms of the quotient group $Q\left(\alpha_{1}, \alpha_{2}, \alpha_{3}\right)$ of $\pi_{1} K$ we worked with in 1.2 , this is a 
matter of one triangle group $Q\left(\alpha_{1}, \alpha_{2}, \alpha_{3}\right)$ containing another triangle group as a subgroup. Plotnick showed that if we assume $\alpha_{1}^{-1}+\alpha_{2}^{-1}+\alpha_{3}^{-1}<1$ (i.e. $Q\left(\alpha_{1}, \alpha_{2}, \alpha_{3}\right)$ is a hyperbolic triangle group), then $Q\left(\alpha_{1}, \alpha_{2}, \alpha_{3}\right)$ contains only a finite number of subgroups which are isomorphic to triangle groups. This implies that repeated applications of our scheme of passing to covers corresponding to hyperelementary subgroups of a finite, nonhyperelementary quotient of $\pi_{1}$ will soon bring us to a situation in which all of the covering spaces of $K^{3}$ under consideration are Haken manifolds. The corresponding covers of $M^{n+3}$ are total spaces of bundles with fiber $N^{n}$ over these Haken manifolds, so 1.1 applies to them to make their Whitehead groups vanish. Repeated applications of 1.3 now show that $\mathrm{Wh}\left(\pi_{1} M\right)=0$, concluding the proof of 1.4 .

2. Structure sets. To Hypothesis A we add the requirement that our fibers $N^{n}$ satisfy this version of the conjecture that homotopy equivalence should imply homeomorphism:

HYPOTHESIS B. $S_{\text {TOP }}\left(N^{n} \times D^{k}, \partial\left(N \times D^{k}\right)\right)=0$ if $k$ is large enough.

Closed manifolds whose fundamental groups are poly-Z groups and complete Riemannian flat manifolds are known to satisfy this condition. The next result reduces the dimension of the disk above.

The corrected version of Siebenmann's periodicity theorem for topological surgery [15, p. 277] found in [17] shows that we can deduce $S_{\mathrm{TOP}}(M, \partial M)=0$ from $S_{\text {TOP }}\left(M \times D^{4}, \partial\left(M \times D^{4}\right)\right)=0$ :

Proposition 2.1. Let $M^{m}$ be a compact, oriented manifold with nonempty boundary. Suppose $m \geqslant 6$. Then $S_{\mathrm{TOP}}(M, \partial M) \cong S_{\mathrm{TOP}}\left(M \times D^{4}, \partial\left(M \times D^{4}\right)\right)$.

Let $M^{m}$ be a closed, oriented manifold and suppose $m \geqslant 5$. Then there is an exact sequence of groups

$$
0 \rightarrow S_{\mathrm{TOP}}(M, \partial M) \rightarrow S_{\mathrm{TOP}}\left(M \times D^{4}, \partial\left(M \times D^{4}\right)\right) \rightarrow L_{0}(1) .
$$

Since $L_{0}(1)=\mathbf{Z}, S_{\mathrm{TOP}}\left(M \times D^{4}, \partial\left(M \times D^{4}\right)\right)$ must be isomorphic either to $S_{\text {TOP }}(M, \partial M)$ or to $S_{\text {TOP }}(M, \partial M) \oplus \mathbf{Z}$; both cases occur.

Recall that the long exact sequence of topological surgery is an exact sequence of groups when $[X, \partial X ; G / \mathrm{TOP}, *]$ is given the "characteristic variety" addition $[17,20,21]$, and that the surgery invariant $\left[X^{n}, \partial X ; G /\right.$ TOP, $\left.*\right] \rightarrow L_{n}\left(\pi_{1} X\right)$ is identified by Poincaré-Lefschetz duality with a natural transformation from the homology theory with coefficients in an $\Omega$-spectrum $\underline{\mathbf{L}}_{0}$, whose 0 th term is homotopy equivalent to $G /$ TOP, to $L$-theory: $\sigma: h_{n}(X) \rightarrow L_{n}\left(\pi_{1} X\right)$. (This paper uses $h_{n}(X)$ for $H_{n}\left(X ; \underline{\mathbf{L}}_{0}\right)$.) This natural transformation is defined for any connected $\mathrm{CW}$-complex $X$ and the long exact sequence of surgery in the topological category is identified with the homotopy sequence of a fibration, with $S_{\mathrm{TOP}}\left(X^{n} \times D^{k}, \partial\left(X \times D^{k}\right)\right)$ identified with $\pi_{n+k+1}$ of the fiber. Note that the statement

$$
\text { “ } S_{\mathrm{TOP}}\left(M^{m} \times D^{k}, \partial\left(M \times D^{k}\right)\right)=0 \text { for } k \text { large enough" }
$$

is equivalent to the statement

$$
\text { “ } \sigma: h_{n}\left(M^{m}\right) \rightarrow L_{n}\left(\pi_{1} M\right) \text { is an isomorphism if } n \text { is large enough." }
$$


If $X^{n}$ is a connected submanifold of $Y^{n+1}$ with trivial normal (micro)bundle such that the inclusion $X \hookrightarrow Y$ induces an injection of fundamental groups, and if $f$ : $V^{n+1} \rightarrow Y^{n+1}$ is a homotopy equivalence, then $f$ is said to split along $X$ if $f$ is homotopic to a map $g$ such that

(a) $g$ is transverse to $X$ (so $W=g^{-1}(X)$ is a codimension-one submanifold of $V$ ), and

(b) the restriction of $g$ to $W, W \rightarrow X$, and to $g^{-1}(Y \backslash X) \rightarrow Y \backslash X$ are homotopy equivalences.

(If $\partial Y \neq \varnothing$ then we ask that $f / \partial V$ be a homotopy equivalence of $\partial V$ to $\partial Y$ already satisfying (a) and (b) with respect to $X$.)

If $Y^{n+1}$ has such a submanifold $X^{n}$ then either

(1) $Y^{n+1}$ is the result of pasting two manifolds $Y_{1}^{n+1}, Y_{2}^{n+1}$ together along $X^{n} \subseteq \partial Y_{j}^{n+1}(j=1,2)$ and $\pi_{1} Y=\pi_{1} Y_{1}{ }_{\pi_{1}}{ } \pi_{1} Y_{2}$, or

(2) $Y^{n+1}$ is the result of identifying two copies of $X^{n}$ in the boundary of a connected manifold $Y_{0}^{n+1}$ and $\pi_{1} Y=\pi_{1} Y_{0}{ }_{\pi_{1} X}$, an HNN construction. Splitting theorems lead to exact sequences for structure sets:

LEMmA 2.2. Suppose $n \geqslant 5,\left(Y^{n+1}, \partial X^{n}\right)$ is a manifold pair as above, $\mathrm{Wh}\left(\pi_{1} Y\right)=$ $\mathrm{Wh}\left(\pi_{1} X\right)=0$, and all homotopy equivalences of pairs of manifolds $\left(V^{n+1}, \partial V\right) \rightarrow$ $\left(Y^{n+1}, \partial Y\right)$ split along $X$. Then we obtain an exact sequence of pointed sets:

(1) if $Y \backslash X$ has two components then there is an exact sequence

$$
S_{\mathrm{TOP}}\left(Y_{1}, \partial Y_{1}\right) \times S_{\mathrm{TOP}}\left(Y_{2}, \partial Y_{2}\right) \rightarrow S_{\mathrm{TOP}}(Y, \partial Y) \rightarrow S_{\mathrm{TOP}}(X, \partial X) ;
$$

(2) if $Y \backslash X$ is connected then there is an exact sequence

$$
S_{\mathrm{TOP}}\left(Y_{0}, \partial Y_{0}\right) \rightarrow S_{\mathrm{TOP}}(Y, \partial Y) \rightarrow S_{\mathrm{TOP}}(X, \partial X) .
$$

Here $Y_{0}, Y_{1}, Y_{2}$ are the manifolds obtained by cutting $Y$ open along $X$. In either case the first arrow is defined by "glue along $X$ " and the second arrow is "split along $X$." The proof of this lemma will not be given here; it may be found in [25] and is implicit in the work of Farrell and Hsiang, and Burghelea.

Cappell [4] showed that all homotopy equivalences to $Y^{n+1}$ split along $X^{n}$ if $\pi_{1} X$ injects into $\pi_{1} Y$ as a square-root closed subgroup, i.e. if $j: X \hookrightarrow Y$ induces $j_{\#}$ : $\pi_{1} X \hookrightarrow \pi_{1} Y$ such that $g \in \pi_{1} Y$ and $g^{2} \in j_{\#}\left(\pi_{1} X\right)$ imply $g \in j_{\#}\left(\pi_{1} X\right)$, subject to the dimension restriction $n \geqslant 5$ and some $K$-theoretic conditions. This splitting theorem, the results of $\S 1,2.1$ and 2.2 yield the next two lemmas.

LEMMA 2.3. If $N^{n} \rightarrow M^{n+2} \rightarrow K^{2}$ is a bundle where $N, M$ and $K$ are orientable, compact, connected manifolds, $N$ satisfies Hypotheses $\mathrm{A}$ and $\mathrm{B}, \partial N=\varnothing, K \neq S^{2}$, and either $n+k \geqslant 4$ or $\partial K=\varnothing$ and $n \geqslant 3$, then $S_{\mathrm{TOP}}\left(M \times D^{k}, \partial\left(M \times D^{k}\right)\right)=0$.

LEMMA 2.4. If $N^{n} \rightarrow M^{n+3} \rightarrow K^{3}$ is a fiber bundle where $N, M$ and $K$ are orientable, compact, connected manifolds, $\partial N=\varnothing, N$ satisfies Hypotheses A and B, and $K^{3}$ is a Haken manifold so that either

(a) $K^{3}$ fibers over $S^{1}$,

(b) $K^{3}$ is a Seifert manifold $M\left(-; g, d ;\left(\alpha_{1}, \beta_{1}\right), \ldots,\left(\alpha_{m}, \beta_{m}\right)\right)$ with nonempty boundary, or 
(c) $K^{3}=M\left(b ; g, 0 ;\left(\alpha_{1}, \beta_{1}\right), \ldots,\left(\alpha_{m}, \beta_{m}\right)\right)$ is a closed manifold with only odd isotropy invariants $\alpha_{i}$.

If $n+k \geqslant 3$ or if $\partial K=\varnothing, k=0$, and $n=2$, then, $S_{\mathrm{TOP}}\left(M \times D^{k}, \partial\left(M \times D^{k}\right)\right)$ $=0$.

As each Seifert manifold with nonempty boundary fibers over the circle, (a) implies (b). The discussion of incompressible surfaces in Seifert manifolds in [14, $\mathrm{p}$. 107] implies that those manifolds $K^{3}=M\left(b ; g, 0 ;\left(\alpha_{1}, \beta_{1}\right), \ldots,\left(\alpha_{m}, \beta_{m}\right)\right)$ which have at least one even isotropy invariant $\alpha_{i}$ have no incompressible surfaces whose fundamental groups inject into $\pi_{1} K^{3}$ as square-root closed subgroups, so we can go no farther with the splitting theorem [4]. Cappell's identification of the obstructions to splitting a homotopy equivalence in $[1,2,3,5]$ gives the following statements:

Lemma 2.5. (a) If $N^{n} \rightarrow M^{n+3} \rightarrow K^{3}$ is a fiber bundle with $N, M$ and $K$ compact, connected, orientable manifolds, $\partial N=\varnothing, N$ satisfies Hypotheses $\mathrm{A}$ and $\mathrm{B}$, and $K^{3}$ is $a$ Haken manifold, then $S_{\mathrm{TOP}}\left(M \times D^{k}, \partial\left(M \times D^{k}\right)\right) \otimes Z[1 / 2]=0$ provided that $n+$ $k \geqslant 3$ or $\partial K=\varnothing, k=0$ and $n=2$.

(b) In fact, under the same hypotheses, $S_{\mathrm{TOP}}\left(M \times D^{k}, \partial\left(M \times D^{k}\right)\right)$ is isomorphic to $\mathrm{UNil}_{n+k+4}(\Phi)$ for any triad $\Phi$ of fundamental groupoids giving $\pi_{1} M$ and induced by an incompressible surface in $K^{3}$, provided $n+k \geqslant 4$.

The groups $\mathrm{UNil}_{*}(-)$ are Cappell's counterparts in $L$-theory to the Waldhausen Nil groups for $K$-theory; they are splitting obstructions and also obstruct the exactness of Mayer-Vietoris sequences in $L$-theory. The UNils are not very well understood, but they are known to be 2-primary groups. 2.5(b) shows that the vanishing statement we are pursuing amounts to a calculation of some of these groups.

Proposition 2.6. If $N^{n} \rightarrow M^{n+3} \rightarrow K^{3}$ is a fiber bundle with $N, M$ and $K$, compact, connected, orientable manifolds, $\partial N=\varnothing, N$ satisfies Hypotheses A and $\mathrm{B}, K^{3}$ is a closed Seifert manifold $M\left(b ; g, 0 ;\left(\alpha_{1}, \beta_{1}\right), \ldots,\left(\alpha_{m}, \beta_{m}\right)\right)$ with $g=$ genus $\left(K^{3} / S^{1}\right) \geqslant 1$, and $n+k \geqslant 3$ or $n \geqslant 2$, then $S_{\mathrm{TOP}}\left(M \times D^{k}, \partial\left(M \times D^{k}\right)\right)=0$.

Proof. Because $\Sigma=K^{3} / S^{1}$ is a closed surface of positive genus, it has an infinite cyclic covering $\Sigma^{\prime} \rightarrow \Sigma$. The circle action on $K$ lifts to an action on the corresponding infinite cyclic cover $K^{\prime}$ of $K$, with $K^{\prime} / S^{1}=\Sigma^{\prime}$. Because $K^{\prime}$ is an open Seifert manifold whose Seifert invariants just repeat those of $K, K^{\prime}$ admits an $S^{1}$. equivariant map to $S^{1}$ and therefore fibers over the circle [6] with an open surface $H$ as fiber. This gives the following structure for $\pi_{1} K$ :

$$
\pi_{1} K=\left(F \rtimes_{\alpha} T\right) \rtimes_{\beta} T
$$

where $F=\pi_{1} H$ is a free group and $T$ is the infinite cyclic group. (Another proof that $\pi_{1} K$ has this structure is given in [25].) The covering and fibering for $K$ induce a covering and fibering for $M$, and a similar structure for $\pi_{1} M$ results:

$$
\pi_{1} M=\left(G \rtimes_{\gamma} T\right) \rtimes_{\delta} T
$$

where $G$, the fundamental group of the induced fiber bundle over the open surface $H$, is given by an extension $1 \rightarrow \pi_{1} N \rightarrow G \rightarrow F \rightarrow 1$. 
Recall that the vanishing we want for $S_{\mathrm{TOP}}$ is equivalent to $\sigma: h_{j} \rightarrow L_{j}$ being an isomorphism for $j$ large enough.

If $H$ is a surface of finite type, then 2.3 shows that $\sigma: h_{j}(K(G, 1)) \rightarrow L_{j}(G)$ is an isomorphism for $j$ sufficiently large (say $j \geqslant m_{0}$ ). Since direct limits commute with homology theories and the $L$-groups, we obtain the same result for our open surface $H$ in general.

Ranicki [21] established a Mayer-Vietoris sequence for the $L$-theory of twisted Laurent extensions $\pi \rtimes T$ which is valid even if the group $\pi$ is not finitely generated. This sequence is natural with respect to the analogous sequence in $h_{*}$ and the surgery homomorphism $\sigma: h_{*} \rightarrow L_{*}$. As $\$ 1$ established that the Whitehead groups of all the groups under consideration here vanish, Ranicki's sequence and the surgery homomorphism give us two ladders:

$$
\begin{array}{ccccccccc}
\rightarrow & h_{j+1}\left(K\left(G \rtimes_{\gamma} T, 1\right)\right) & \rightarrow & h_{j}(K(G, 1)) & \rightarrow & h_{j}(K(G, 1)) & \rightarrow & h_{j}\left(K\left(G \rtimes_{\gamma} T, 1\right)\right) & \rightarrow \\
& \downarrow & & & \downarrow & & \downarrow & & \downarrow \\
& L_{j+1}\left(G \rtimes_{\gamma} T\right) & \rightarrow & L_{j}(G) & \rightarrow & L_{j}(G) & \rightarrow & L_{j}\left(G \rtimes_{\gamma} T\right) & \rightarrow
\end{array}
$$

and

$$
\begin{aligned}
& \rightarrow h_{j+1}(M) \rightarrow h_{j}\left(K\left(G \rtimes_{\gamma} T, 1\right)\right) \rightarrow h_{1}\left(K\left(G \rtimes_{\gamma} T, 1\right)\right) \rightarrow h_{j}(M) \rightarrow
\end{aligned}
$$

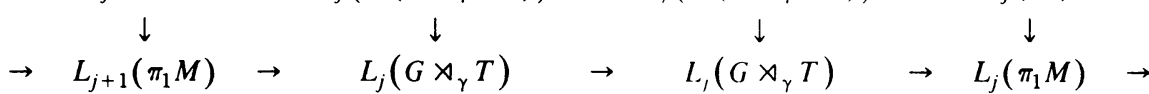

The Five Lemma applies to these ladders to show that $\sigma: h_{j}\left(K\left(G \rtimes_{\gamma} T, 1\right)\right) \rightarrow$ $L_{j}\left(G \rtimes_{\gamma} T\right)$ is an isomorphism for $j \geqslant m_{0}+1$ and that $\sigma: h_{j}(M) \rightarrow L_{j}\left(\pi_{1} M\right)$ is an isomorphism for $j \geqslant m_{0}+2$ (recall that $\left.M=K\left(G \rtimes_{\gamma} T\right) \rtimes_{\delta} T, 1\right)$ ). Apply Proposition 2.1 to obtain the claimed dimensions for this vanishing statement, whatever $m_{0}$ might be.

This completes the proof of 2.6.

3. Some structure sets when $K^{3} / S^{1}=S^{2}$. In many of these cases the only difficulties are 2-local:

LEMMA 3.1. Let $N^{n} \rightarrow M^{n+3} \rightarrow K^{3}$ be a fiber bundle with $N, M$ and $K$ compact, connected, orientable manifolds, $\partial N=\varnothing$, where $N$ satisfies Hypotheses $\mathrm{A}$ and $\mathrm{B}$, and $K^{3}=M\left(b ; 0,0 ;\left(\alpha_{1}, \beta_{1}\right), \ldots,\left(\alpha_{m}, \beta_{m}\right)\right)$ is a Seifert manifold with base $S^{2}$. Suppose $K^{3}$ is sufficiently large or $m=3$ and $\alpha_{1}^{-1}+\alpha_{2}^{-1}+\alpha_{3}^{-1}<1$. Then

$$
S_{\mathrm{TOP}}\left(M \times D^{k}, \partial\left(M \times D^{k}\right)\right) \otimes Z[1 / 2]=0
$$

provided that $n+k \geqslant 3$ or $n=2$ and $k=0$.

When $K^{3}$ is sufficiently large this is Lemma 2.5. The remaining cases, in which the group $\pi_{1} K / j_{\#}\left(\pi_{1} S^{1}\right)$ is a hyperbolic triangle group, are dealt with by following Plotnick's induction scheme from $\$ 1$ and applying part (3) of the following consequence of the induction theorems of Nicas [17] (see especially Proposition 6.2.9).

Proposition 3.2. Suppose $M^{m}$ is a compact manifold and $m \geqslant 5$ ( $m \geqslant 6$ if $\partial M \neq \varnothing)$. Let $f: \pi_{1} M \rightarrow G$ be an epimorphism onto a finite group, and for each subgroup $H$ of $G$ let $M_{H}$ be the covering space corresponding to $f^{-1}(H)<\pi_{1} M$. 
(1) If $S_{\mathrm{TOP}}\left(M_{H} \times D^{k}, \partial\left(M_{H} \times D^{k}\right)\right) \otimes \mathbf{Q}=0$ for each cyclic subgroup $H$ of $G$ then $S_{\mathrm{TOP}}\left(M \times D^{k}, \partial\left(M \times D^{k}\right)\right) \otimes \mathbf{Q}=0$.

(2) If $S_{\mathrm{TOP}}\left(M_{H} \times D^{k}, \partial\left(M_{H} \times D^{k}\right)\right) \otimes \mathbf{Z}_{(2)}=0$ for each 2-hyperelementary subgroup $H$ of $G$ then $S_{\mathrm{TOP}}\left(M \times D^{k}, \partial\left(M \times D^{k}\right)\right) \otimes \mathbf{Z}_{(2)}=0$. (Recall that $\mathbf{Z}_{(2)}=$ $\mathbf{Z}[1 / 3,1 / 5, \ldots]$.)

(3) If $S_{\mathrm{TOP}}\left(M_{H} \times D^{k}, \partial\left(M_{H} \times D^{k}\right)\right) \otimes \mathbf{Z}[1 / 2]=0$ for each -elementary subgroup $H$ of $G$ and each odd prime $p$ then $S_{\mathrm{TOP}}\left(M \times D^{k}, \partial\left(M \times D^{k}\right)\right) \otimes \mathbf{Z}[1 / 2]=0$.

(4) If $S_{\mathrm{TOP}}\left(M_{H} \times D^{k}, \partial\left(M_{H} \times D^{k}\right)\right)=0$ for each subgroup $H$ of $G$ in the union of the three classes of subgroups above then $S_{\mathrm{TOP}}\left(M \times D^{k}, \partial\left(M \times D^{k}\right)\right)=0$.

From part (2) of the proposition we can show that structure sets vanish for the manifolds of Lemma 3.1 by studying the covers corresponding to 2-hyperelementary subgroups $H$ of a finite quotient $G$ of $\pi_{1} K^{3}$. The results in $\$ 2$ give two "won positions" for such a cover $K_{H}$ of $K$ :

(a) If $K_{H}$ is sufficiently large and all its isotropy invariants are odd then the corresponding cover $M_{H}$ of $M$ has $S_{\mathrm{TOP}}\left(M_{H} \times D^{k}, \partial\left(M_{H} \times D^{k}\right)\right)=0$. (This is 2.4(c).)

(b) If $K_{H}$ has genus $\left(K_{H} / S^{1}\right) \geqslant 1$ then the corresponding cover $M_{H}$ of $M$ has $S_{\text {TOP }}\left(M_{H} \times D^{k}, \partial\left(M_{H} \times D^{k}\right)\right)=0$. (This is 2.6.)

If each isotropy invariant $\alpha_{i}$ of $K^{3}=M\left(b ; 0,0 ;\left(\alpha_{1}, \beta_{1}\right),\left(\alpha_{2}, \beta_{2}\right),\left(\alpha_{3}, \beta_{3}\right)\right)$ is odd then the isotropy invariants of any Seifert manifold $K_{H}$ covering $K S^{1}$-equivariantly will also be odd. Thus 1.2, 2.4(c) and 3.2 give us a vanishing statement for some of the insufficiently large examples:

COROLlaRY 3.3. If $N^{n} \rightarrow M^{n+3} \rightarrow K^{3}$ is a fiber bundle as in Lemma 3.1 and $K^{3}=M\left(b ; 0,0 ;\left(\alpha_{1}, \beta_{1}\right),\left(\alpha_{2}, \beta_{2}\right),\left(\alpha_{2}, \beta_{3}\right)\right)$ with $\alpha_{1}^{-1}+\alpha_{2}^{-1}+\alpha_{3}^{-1}<1$ and each $\alpha_{i}$ odd, then $S_{\mathrm{TOP}}\left(M \times D_{k}, \partial\left(M \times D^{k}\right)\right)=0$ provided that $n+k \geqslant 3$ or $n=2$ and $k=0$.

The next result makes use of the second class of won positions.

Proposition 3.4. If $N^{n} \rightarrow M^{n+3} \rightarrow K^{3}$ is a fiber bundle as in Lemma 3.1 and $K^{3}=M\left(b ; 0,0 ;\left(\alpha_{1}, \beta_{1}\right),\left(\alpha_{2}, \beta_{2}\right), \ldots,\left(\alpha_{m}, \beta_{m}\right)\right)$ where an odd prime $p$ divides one of the $\alpha_{j}$ 's, say $\alpha_{1}$, and the group $Q\left(\alpha_{1} / p, \alpha_{2}, \ldots, \alpha_{m}\right)$ is hyperbolic, then

$$
S_{\mathrm{TOP}}\left(M \times D^{k}, \partial\left(M \times D^{k}\right)\right)=0
$$

provided that $n+k \geqslant 3$ or $n=2$ and $k=0$.

Proof. Imitating the argument for 1.4, we iterate the operation of taking a finite quotient $G$ of a manifold's fundamental group and passing to the covering spaces corresponding to 2-hyperelementary subgroups $H$ of $G$. The reader might wish to think of this process as constructing a tree of covering spaces beginning with $M^{n+3}$, where a level of the tree consists of covering spaces of the manifolds in the next lower level, with each covering associated to a 2-hyperelementary subgroup of a finite quotient of $\pi_{1}$ of the base of that covering projection, and where the goal is to produce a tree whose topmost branches all represent manifolds with vanishing structure sets. 
We will rely on two facts concerning products of finite cyclic groups.

FACT 1. If $p$ is an odd prime and $T_{p}$ is the cyclic group of order $p$, then each 2-hyperelementary subgroup $H$ of $\left(T_{p}\right)^{s}=T_{p} \times \cdots \times T_{p}$ (s factors) is isomorphic to $T_{p}$.

FACT 2. If $p$ is an odd prime and $X$ is a cohomology $n$-sphere over $\mathbf{Z} / p$ with an effective $\left(T_{p}\right)^{s}$-action, then $s \leqslant(n+1) / 2$.

The first fact follows immediately from the definition of a 2-hyperelementary group. The second fact is a result of Smith theory [24]. Note that the second fact says $T_{p} \times T_{p}$ cannot act effectively on the 2 -sphere.

Let $Q=Q\left(\alpha_{1}, \ldots, \alpha_{m}\right)$ be the quotient group $\pi_{1} K^{3} / j_{\#}\left(\pi_{1} S^{1}\right)$.

LEMMA 3.5. If $M$ is as in the statement of the proposition and if $Q$ admits an epimorphism $h: Q \rightarrow\left(T_{p}\right)^{s}$ with $p$ an odd prime and $s \geqslant 3$, then

$$
S_{\mathrm{TOP}}\left(M \times D^{k}, \partial\left(M \times D^{k}\right)\right)=0
$$

if $n+k \geqslant 3$ or if $k=0$ and $n=2$.

Proof of LEMma. Let $H$ be a 2-hyperelementary subgroup of $\left(T_{p}\right)^{s}$. Then $H \approx T_{p}$ and $H$ is normal in $\left(T_{p}\right)^{s}$, so $h^{-1}(H)$ is a normal subgroup of $Q$ with $Q / h^{-1}(H)=$ $\left(T_{p}\right)^{s} / H=\left(T_{p}\right)^{s-1}$. This means that the corresponding branched covering $R H^{2} / h^{-1}(H) \rightarrow R H^{2} / Q$ is a regular branched cover with automorphism group $\left(T_{p}\right)^{s-1}$. As $s-1 \geqslant 2,\left(T_{p}\right)^{s-1}$ cannot act effectively on $S^{2}$, so genus $\left(R H^{2} / h^{-1}(H)\right)$ $\geqslant 1$, i.e., the cover of $K^{3}$ corresponding to $H$ via $\pi_{1} K^{3} \rightarrow Q \stackrel{h}{\rightarrow}\left(T_{p}\right)^{s}$ is a Seifert manifold over a surface of positive genus. The conclusion follows from 2.6 and 3.2.

If an odd prime $p$ divides at least four of the isotropy invariants $\alpha_{i}$ then $Q$ has an explicit epimorphism to $\left(T_{p}\right)^{3}$. Suppose $p$ divides $\alpha_{1}, \alpha_{2}, \alpha_{3}$ and $\alpha_{4}$. $Q$ has the presentation

$$
Q=\left\langle q_{1}, q_{2}, \ldots, q_{m}: q_{1}^{\alpha_{1}}=q_{2}^{\alpha_{2}}=\cdots=q_{m}^{\alpha_{m}}=q_{1} q_{2} \cdots q_{m}=1\right\rangle .
$$

Give $\left(T_{p}\right)^{3}$ the presentation

$$
\left(T_{p}\right)^{3}=\left\langle t_{1}, t_{2}, t_{3}: t_{j}^{p}=\left[t_{i}, t_{j}\right]=1\right\rangle
$$

and define a homomorphism $h: Q \rightarrow\left(T_{p}\right)^{3}$ by

$$
\begin{aligned}
& q_{i} \mapsto t_{i} \quad(i=1,2,3), \\
& q_{4} \mapsto\left(t_{1} t_{2} t_{3}\right)^{-1}, \\
& q_{j} \mapsto 1 \quad(j \geqslant 5) .
\end{aligned}
$$

This observation and 3.5 reduce our problem to producing a tree of covers whose topmost branches have enough isotropy invariants divisible by an odd prime $p$.

Suppose $\alpha_{1}$ is divisible by the odd prime $p$, and

$$
Q\left(\alpha_{1}, \alpha_{2}, \ldots, \alpha_{m}\right) \text { and } Q\left(\alpha_{2}, \ldots, \alpha_{m}\right)
$$

are both hyperbolic groups. Then there is an epimorphism $f: Q\left(\alpha_{1}, \alpha_{2}, \ldots, \alpha_{m}\right) \rightarrow$ $Q\left(\alpha_{2}, \ldots, \alpha_{m}\right)$,

$$
q_{1} \mapsto 1, \quad q_{j} \mapsto q_{j} \quad(2 \leqslant j \leqslant m),
$$


and we know there is an epimorphism $h: Q\left(\alpha_{2}, \ldots, \alpha_{m}\right) \rightarrow G$, where $G$ is a finite, non-2-hyperelementary group. Let $H$ run over the 2-hyperelementary subgroups of $G$ and consider the subgroups $h^{-1}(H)$ of $Q\left(\alpha_{2}, \ldots, \alpha_{m}\right)$. For each $h^{-1}(H)$ repeat the process of taking a non-2-hyperelementary finite quotient and subgroups corresponding to the 2-hyperelementary subgroups of that quotient. Via $f$ this process pulls back to $Q\left(\alpha_{1}, \alpha_{2}, \ldots, \alpha_{m}\right)$ to construct a two-stage tree of covers of $K^{3}$. Since each subgroup has index 2 or more in the group just below it in the tree, each subgroup $S$ at the top of the tree has index 4 or more in $Q\left(\alpha_{1}, \alpha_{2}, \ldots, \alpha_{m}\right)$. This implies that in each of these subgroups $S$ there are at least 4 elements of order $\alpha_{1}$ which are not $S$-conjugate. To see this it helps to think of the geometry associated to this system of groups: each group is associated to a closed Riemann surface with elliptic points corresponding to the elliptic elements (that is, the torsion elements), modulo conjugacy. On the surface corresponding to $Q\left(\alpha_{2}, \ldots, \alpha_{m}\right)$ one can imagine a freckle corresponding to the forgotten elliptic point of the surface associated to $Q\left(\alpha_{1}, \alpha_{2}, \ldots, \alpha_{m}\right)$. Since the freckle is not a branch point for any of the branched covers corresponding to subgroups of $Q\left(\alpha_{2}, \ldots, \alpha_{m}\right)$, it is just copied over in each of those branched covers, and the number of copies of this freckle in the source surface is given by the degree of the map (i.e. the index of the subgroup in $Q\left(\alpha_{2}, \ldots, \alpha_{m}\right)$ ). Since each subgroup of $S$ of $Q\left(\alpha_{1}, \alpha_{2}, \ldots, \alpha_{m}\right)$ at the top of the tree has at least four nonconjugate elements whose orders are divisible by $p$, the present case reduces to that successfully dealt with in Lemma 3.5.

If $Q$ is a Fuchsian group with no punctures, genus $g$, and $m$ elliptic elements of orders $\alpha_{1}, \alpha_{2}, \ldots, \alpha_{m}$, let $a(Q)=2 g-2+\sum_{1 \leqslant j \leqslant m}\left(1-\alpha_{j}^{-1}\right)$. If $Q_{1}$ is a subgroup of index $d$ in $Q$ then the Riemann-Hurwitz theorem asserts that $a\left(Q_{1}\right)=d \cdot a(Q)$. Note that our group $Q\left(\alpha_{1}, \ldots, \alpha_{m}\right)$ is Fuchsian (i.e. hyperbolic) if and only if $a\left(Q\left(\alpha_{1}, \ldots, \alpha_{m}\right)\right)=-2+\sum_{1 \leqslant j \leqslant m}\left(1-\alpha_{j}^{-1}\right)$ is positive.

Consider now the situation in the statement of the proposition: an odd prime $p$ divides $\alpha_{1}$ and the groups $Q\left(\alpha_{1}, \alpha_{2}, \ldots, \alpha_{m}\right)$ and $Q\left(\alpha_{1} / p, \alpha_{2}, \ldots, \alpha_{m}\right)$ are hyperbolic. Choose a positive integer $r$ so that $2^{r} \cdot a\left(Q\left(\alpha_{1}, \ldots, \alpha_{m}\right)\right)>1-p^{-1}$. Construct a tree of coverings of $K^{3}$ with $r$ levels by applying the usual recipe to $Q\left(\alpha_{1} / p, \alpha_{2}, \ldots, \alpha_{m}\right)$ $r$ times and by using the obvious epimorphism

$$
Q\left(\alpha_{1}, \alpha_{2}, \ldots, \alpha_{m}\right) \rightarrow Q\left(\alpha_{1} / p, \alpha_{2}, \ldots, \alpha_{m}\right) .
$$

(If at some intermediate level in the tree one of the cases previously resolved appears, then the tree need not grow further along that branch.) The uppermost covering spaces in this tree of covers of $K^{3}$ correspond to subgroups $S$ of $Q\left(\alpha_{1}, \alpha_{2}, \ldots, \alpha_{m}\right)$ with the following properties.

(a) $S$ contains at least one elliptic generator $q_{*}$ whose order $\alpha_{*}$ is divisible by $p$.

(b) $a(S)>1-p^{-1}$. Since $1-p^{-1} \geqslant 1-\alpha_{*}^{-1}$ it follows that the group obtained from $S$ by identifying the generator $q_{*}$ to 1 is hyperbolic. Therefore each of these subgroups $S$ is, at worst, of the sort considered two paragraphs previously. This final reduction to known cases completes the proof of the proposition.

\section{REFERENCES}

1. S. E. Cappell, Mayer-Vietoris sequences in Hermitian K-theory, Algebraic $K$-theory. III, Lecture Notes in Math., vol. 343, Springer-Verlag, Berlin and New York, 1973, pp. 478-512.

2. U_ Unitary nilpotent groups and Hermitian K-theory. I, Bull. Amer. Math. Soc. 80 (1974), 1117-1122. 
3. (1974), 1193-1198.

4. __ A splitting theorem for manifolds, Invent. Math. 33 (1976), 69-170.

5. __ On homotopy invariance of higher signatures, Invent. Math. 33 (1976), 171-179.

6. P. E. Conner and F. Raymond, Injective actions of the toral groups, Topology 10 (1971), 283-296.

7. F. T. Farrell and W. C. Hsiang, $A$ formula for $K_{1} R_{\alpha}[T]$, Proc. Sympos. Pure Math., vol. 17, Amer. Math. Soc., Providence, R. I., 1970, pp. 192-218.

8. __ The topological-Euclidean space form problem, Invent. Math. 45 (1978), 181-192.

9. 308-324.

10. Topological characterization of flat and almost flat Riemannian manifolds $M^{n}(n \neq 3,4)$,

Amer. J. Math. 105 (1983), 641-672.

11. R. H. Fox, On Fenchel's conjecture about F-groups, Mat. Tidsskr. B 1952 (1952), 61-65.

12. J. Hempel, 3-Manifolds, Princeton Univ. Press, Princeton, N. J., 1976.

13. Homology of coverings, preprint.

14. W. Jaco, Lectures on three-manifold topology, CBMS Regional Conf. Ser. in Math.,vol. 43, Amer. Math. Soc., Providence, R. I., 1980.

15. R. C. Kirby and L. C. Siebenmann, Foundational essays on topological manifolds, smoothings, and triangulations, Princeton Univ. Press, Princeton, N. J., 1977.

16. R. C. Lyndon and P. E. Schupp, Combinatorial group theory, Springer-Verlag, Berlin and New York, 1977.

17. A. J. Nicas, Induction theorems for groups of homotopy manifold structures, Mem. Amer. Math. Soc. No. 267 (1982).

18. P. Orlik, Seifert manifolds, Lecture Notes in Math., vol. 291, Springer-Verlag, Berlin and New York, 1972.

19. S. Plotnick, Vanishing of Whitehead groups for Seifert manifolds with infinite fundamental group, Comment. Math. Helv. 55 (1980), 654-667.

20. F. Quinn, A geometric formulation of surgery, Topology of Manifolds (Georgia Conf. 1969), Markham, Chicago, 1970, pp. 500-511.

21. A. Ranicki, Algebraic L-theory. III. Twisted Laurent extension, Algebraic $K$-theory. III, Lecture Notes in Math., vol. 343, Springer-Verlag, Berlin and New York, 1973, pp. 412-463.

22. __ The total surgery obstruction, Algebraic Topology Aarhus 1978, Lecture Notes in Math., vol. 763, Springer-Verlag, Berlin and New York, 1979, pp. 275-316.

23. __ Exact sequences in the algebraic theory of surgery, Princeton Univ. Press, Princeton, N. J., 1981.

24. P. A. Smith, New results and old problems in finite transformation groups, Bull. Amer. Math. Soc. 66 (1960), 401-415.

25. C. W. Stark, Structure sets vanish for certain bundles over Seifert manifolds, Ph. D. Thesis, Univ. of Michigan, 1982.

26. R. Swan, Induced representations and projective modules, Ann. of Math. (2) 71 (1960), 552-578.

27. , K-theory of finite groups and orders, Lecture Notes in Math., vol. 149, Springer-Verlag,

Berlin and New York, 1970 .

28. F. Waldhausen, Whitehead groups of generalized free products, Algebraic $K$-theory. II, Lecture Notes in Math., vol. 342, Springer-Verlag, Berlin and New York, 1973, pp. 155-179.

29. __ Algebraic K-theory of generalized free products, Ann. of Math. (2) 108 (1978), 135-256.

30. C. T. C. Wall, Surgery on compact manifolds, Academic Press, New York and London, 1970.

Department of Mathematics, Brandeis University, Waltham, MassachusetTs 02254 